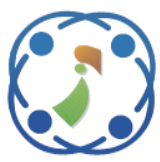

\title{
A Global Sparse Model with Singular Value Decomposition and Huber Loss Function for Rain Streak Removal
}

\author{
Rachapalli Sunanda ${ }^{1 *}$ \\ Joseph Beatrice Seventline ${ }^{1}$ \\ ${ }^{I}$ Gandhi Institute of Technology and Management University, Visakhapatnam, India \\ *Corresponding author's Email: sunanda443@gmail.com
}

\begin{abstract}
The rainy effect normally decreases the visual quality of the images that highly affects the outdoor vision system's performance. Due to light scattering, the rain streaks generate the haziness and blurring effect. So, an effective model is required to remove rain streaks from the single image that assists an extensive range of applications such as object tracking, image enhancement, etc. In this research work, a new model has been proposed for rain streak removal in the single image. In the proposed work, a global sparse model with Singular Value Decomposition (SVD) and Huber loss function were used to remove rain streaks from the synthesized images. In the proposed model, three sparse terms (characteristics of image background information, structural knowledge and intrinsic direction of rain streaks) were used for depicting the directional smoothness of rain-free and rain streak images and also described the intrinsic and latent properties of rain streaks. The rain streaks sparsity was also enhanced by employing 1_1 norm that effectively avoids the undesired features on the rain free regions. Then, Alternating Direction Method of Multipliers (ADMM) was used for tackling the proposed model in order to achieve optimal solutions. In the experimental phase, the proposed model was compared with the existing models like Cascading Attention Aggregation Network (CAAN) and Directional Global Sparse Model (DGSM) in terms of Peak Signal to Noise Ratio (PSNR), Structural Similarity (SSIM), and Root Mean Square Error (RMSE). Through the experimental simulation, the proposed model almost showed 0.52-1 dB improvement in PSNR value.
\end{abstract}

Keywords: Directional global sparse model, Huber loss function, Single image rain removal, Singular value decomposition.

\section{Introduction}

Generally, the performance of outdoor vision systems like autonomous navigation and surveillance is affected by the visual distortions on the image, which is caused due to bad weather conditions [1]. The adverse weather is categorized into two classes (dynamic and steady bad weather) on the basis of constituent particle size in atmosphere [2]. The steady bad weather occurs due to microscopic atmospheric particles like smoke, haze, fog, etc. Correspondingly, the dynamic bad weather condition occurs due to large atmospheric particles like snow, hail, rain, etc. [3, 4]. Thus, the resulting effect of dynamic and steady bad weather conditions are the loss of image details that significantly lessen the performance of outdoor vision applications like event detection, image registration, classification, scene analysis, image retrieval, etc. [5]. Compared to steady bad weather, dynamic bad weather conditions have a huge impact on the outdoor vision systems. Hence, the large atmospheric particles are randomly distributed that leads to complex intensity fluctuations in small image regions. Also, it has a negative impact on the reliability of image feature detection modules of computer vision systems [6]. So, there is a need to develop an automated system to eliminate undesired visual effects from the images for enhancing the reliability of outdoor vision systems [7, 8].

In recent decades, there are numerous systems available to recover original outdoor images from bad weather conditions, especially on rain [9]. Most of the existing systems cause non-rain regions that lead to the loss of image texture information and 
over smoothed, because the background textures and rain streaks are inherently overlapped in the feature space [10]. In order to improve the performance of rain streak removal process, a new system was proposed in this research work. For the experimental analysis, two online datasets (rain100H and rain100L) and a real-time dataset were considered. The proposed work formulates an enhanced Directional Global Sparse Model (DGSM) for rain streak removal in combination with Huber loss function and SVD. In order to preserve the non-rain image pixel details and to remove the rain pixels, the Huber loss function and SVD were incorporated with DGSM. In addition, the proposed model comprises of three sparse terms; characteristics of image background information, structural knowledge and intrinsic direction of rain streaks. Finally, ADMM was applied for solving the proposed convex model that helps in obtaining the global optimal solution. The existing and proposed model's performance was investigated in terms of PSNR, SSIM, and RMSE.

This research paper is presented as follows. In section 2, related research articles on rain streak removal are surveyed. Section 3, explained about the proposed work with mathematical derivations. In section 4, the result and discussion are carried out with graphical comparisons. Finally, the conclusion is presented in the section 5 .

\section{Literature survey}

Y. Luo, and J. Ling [11] presented a new single image deraining methodology based on Low Rank Matrix Approximation (LRMA). The developed method directly leverages the intrinsic characteristics of clear non-rain images without designing hand-crafted rain priors. Also, it formulates the deraining issue as a classical robust Principal Component Analysis (PCA) problem. The experimental consequences illustrate that the developed deraining methodology achieved better performance related to the existing methods on realtime and most synthesized rain images. The major disadvantage of LRMA was not efficient in the rain streak removal process, so the convex nuclear norm was replaced with non-convex surrogates for improving the rain streak removal performance.

D.Y. Chen, C.C. Chen, and L.W. Kang [12] presented a new framework for eliminating rain streaks from the single rainy colour image. At first, guided filter was used for decomposing the collected images into high and low frequency parts. In addition, the high frequency parts were decomposed into non-rain and rain components by utilizing dictionary learning and sparse coding, because the rain streak details would be in high frequency components with non-rain edges. Then, the hybrid features (Eigen color, histogram of oriented gradients, and field depth) were applied to separate rain streaks from the high frequency components. Related to the existing methodologies, the developed framework showed promising outcome in rain streak removal. In this literature, the detection and elimination of rain streaks from the single image will not be precise, if only pixel intensities are involved.

X. Fu, J. Huang, X. Ding, Y. Liao, and J. Paisley [13] used a new deep learning network named as DerainNet for eliminating rain streaks from the collected images. In this literature, the digital images were acquired from the Berkeley segmentation dataset, uncompressed colour image dataset, and a few Google images. Initially, the undertaken network learns the mapping relation between clean and rainy images. In contrast to other approaches, the developed network increases the network depth by adjusting the objective function, which enhances the rain streak elimination performance. Compared to the existing deraining methodologies, the developed network (DerainNet) showed better performance in rain streak removal with faster computation time. Usually, the low resolution images deliver poor results in the DerainNet network, because the low resolution images do not support high level layers.

Z. Shi, Y. Li, C. Zhang, M. Zhao, Y. Feng, and B. Jiang [14] developed a new filtering approach (median guided filter) to remove rain streaks from the collected images. The developed filtering approach contains two filtering operations, (i) weighted median filter was initially applied on the collected images for obtaining coarse rain free images and (ii) guided filter was used for achieving refined rainy free images. In this scenario, the coarse rain free images were utilized as guided images and then convolute the coarse rain free images with collected rainy images utilizing guided filter. Experimental consequences showed that the developed filtering approach attained good results compared to other algorithms. Still, the rain streak removal from a single image is an extremely challenging issue, due to the occurrence of nonuniform rain masses in the images.

C.H. Yeh, C.Y. Lin, K. Muchtar, and P.H. Liu [15] developed Non Negative Matrix Factorization (NNMF) to remove rain streaks from the collected images. Initially, Gaussian filter was employed on the collected images for separating high and low frequency components. Usually, the rain 
components lie in the middle frequency range, which need to be discarded from the low and high frequency domains. In this literature paper, block copy approach and canny edge detection were utilized to eliminate the rain components from the high frequency domains. Then, NNMF method was employed for extracting the rain components from the low frequency domains. The developed framework attained better results in comparison with the existing methodologies. Still, it is hard to determine the angles of rain in the middle frequencies that is considered as one of the major concerns in the developed framework.

L.J. Deng, T.Z. Huang, X.L. Zhao, and T.X. Jiang [16] presented a simple model, named as DGSM for eliminating rain streaks from the single rainy colour image. The developed model includes three sparse terms by considering the features of background image information, the structural knowledge and intrinsic direction of rain streaks. In addition, the ADMM was utilized for achieving the global optimum solution. From the experimental examination, the developed model achieved better results related to the existing rain removal approaches in terms of PSNR and SSIM. Moreover, the heavy rain normally incorporates haze and rain streaks, so the DGSM cannot remove the rain streaks individually.

J. Wang, X. Huang, and S. Gai [17] developed a new algorithm (CAAN) for rain streak removal. At first, the dilated convolution kernels of different scale branches were utilized for extracting the features of dissimilar rain streak sizes. Then, local multi scale residual attention block was used to guide the learned features to be discriminative. The developed cascaded sub network propagates the information from lower to higher layers that decreases the loss of information. At last, the dual channel attention process was utilized to make feature fusion effective. From the experimental simulation, the developed algorithm attained better performance compared to the earlier methods in terms of both image visual quality and quantitative metrics. The CAAN algorithm's performance usually depends on the amount of input data, if the data are fewer than the CAAN algorithm performs poorly.

In order to address the above mentioned concerns, an enhanced DGSM has been proposed for improving the performance of rain streak removal in the single color image.

\section{Methodology}

Generally, the weather conditions like fog, rain, haze, and snow leads to complex visual effects of temporal or spatial domains in the images that considerably lessen the performance of outdoor vision systems and models like object detection, event detection, image registration, tracking, scene analysis, image retrieval, etc. Recently, rain streak elimination from the single image gained more attention within the researcher's community. In this work, a new model was proposed to enhance the performance of rain streak removal in the single images. The detailed explanation about the proposed model is given below.

\subsection{Data collection}

Initially, the input rainy images have been collected from rain $100 \mathrm{H}$ dataset, rain100L dataset and a real time dataset [16]. The rain $100 \mathrm{H}$ dataset comprises of 1800 training images and 200 testing images, which have five rain stripes in dissimilar directions. In addition, the rain100L dataset consists of 200 training images and 100 testing raining images with single style low-density rain streaks [18]. Meanwhile, the real time dataset includes 15 rainy images in that 3 images are synthesized images and the remaining 12 images are collected from http://yu-li.github.io/paper/li_cvpr16_rain.zip

The sample collected rainy images are graphically indicated in Fig. 1.
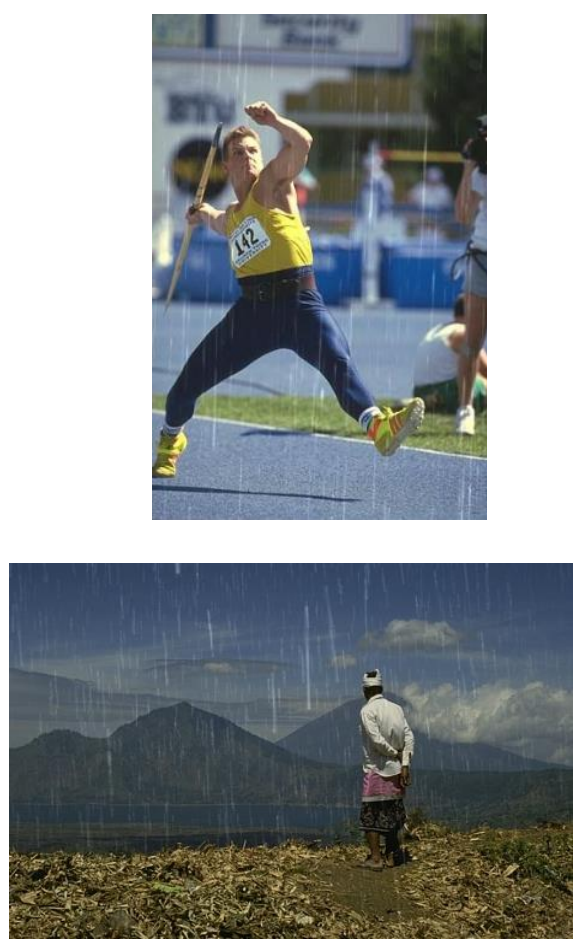

Figure.1 Sample collected images 


\subsection{Proposed model}

\subsubsection{Rain streaks sparsity}

Let us assume the rain streaks being sparse, while the rain is not high. Usually, the rain streaks sparsity is described using $l_{0}$ norm, which is stated as the number of non-zero elements. In this research study, $l_{1}$ norm is utilized for describing the rain streaks sparsity, due to the non-convexity nature of $l_{0}$ norm. The major benefit of using $l_{1}$ norm is its convexity that promises the global optimum in some algorithms like ADMM. Also, the $l_{1}$ norm significantly avoids the undesired features on the rainy free regions. Therefore, the $l_{1}$ norm is utilized as one of the regularizers in this research, which is mathematically indicated in Eq. (1).

$$
\operatorname{Reg}^{(1)}(s)=\|s\|_{1}
$$

Where, $S$ is denoted as rain streaks, and $s$ is indicated as vector version of $S$.

\subsubsection{Rain streaks sparsity along with vertical direction}

Generally, the rain streaks come down from top to bottom in the real scenes of rainfall that is theoretically called as vertical direction (y-direction) of the rainy colour images. Usually, the difference between the adjacent image pixel is small (roughly zero), due to the smoothness within the rain streaks. Additionally, the sparse prior is considered in evaluating the variations in y-direction of the rainy colour images. In some circumstances, the rain streaks do not fall in the vertical direction. So, finding the real direction of rainfall is a very challenging process. In this work, a rotation strategy is utilized for the large angle cases in order to find the real rain-fall direction and angle between the vertical directions. Eq. (2) states the final regularizer assumed for the variation within the rain streaks.

$$
\operatorname{Reg}^{(2)}(s)=\left\|\nabla_{y} s\right\|_{1}
$$

Where, $\nabla_{y}$ is denoted as difference operator in light of the vertical direction, and $\nabla_{y} s$ is stated as vector version of $\nabla_{y} S$.

\subsubsection{Rain streaks sparsity along with horizontal direction}

The variational information in the direction of rain streak is utilized to estimate the discontinuity of rain streak that helps in obtaining the robust rain removal. In this scenario, the across rain streak direction is called as horizontal direction. Due to relationship of $t=r-s$, Eq. (3) indicates the final regularizer assumed for the rain free image across the rain streak direction.

$$
\operatorname{Reg}^{(3)}(s)=\left\|\nabla_{x}(r-s)\right\|_{1}
$$

Where, $\nabla_{x}$ is denoted as difference operator in light of the horizontal direction, and $\nabla_{x}(r-s)$ is indicated as vector version of $\nabla_{x}(R-S), R$ is represented as observed rainy image, and $S$ is stated as rain streaks.

\subsubsection{Non-negative constraint}

Usually, the rain streaks $s$ are non-negative, which have bright pixel intensity in a rainy image. Eq. (4) states the relationship between the rain streaks $s$ and rainy colour images $r$.

$$
r \geq s \geq 0
$$

After investigating the characteristics of rain streaks, find the naïve sparse model along the vertical (y-direction) and horizontal (x-direction) for rain removal problem. Sometimes, the undertaken regularizers seem to be unreasonable, when the direction of rain streaks is far away from the vertical direction $\left(90^{\circ}\right)$. So, a rotation operation $D_{\theta}$ is utilized with the rotation angle $\theta$ for the all three regularizers

$\left\|D_{\theta} s\right\|_{1},\left\|\nabla_{y}\left(D_{\theta} s\right)\right\|_{1}$, and $\left\|\nabla_{x}\left(D_{\theta}(r-s)\right)\right\|_{1}$. Henceforth, the final minimization model to solve the rain removal issue is denoted in Eq. (5).

$$
\min _{s} \lambda_{1}\left\|\nabla_{x}\left(D_{\theta}(r-s)\right)\right\|_{1}+\lambda_{2}\left\|\nabla_{y}\left(D_{\theta} s\right)\right\|_{1}+
$$

Where, $\lambda_{1}$ and $\lambda_{2}$ are indicated as two positive regularization parameters.

\subsection{Proposed model algorithm}

The proposed $l_{1}$ model is not differentiable, so variable substitution is performed to solve the equivalent problem, as mentioned in Eq. (6).

$$
\min \lambda_{1}\|u\|_{1}+\lambda_{2}\|v\|_{1}+\|w\|_{1}
$$

Where, $u=\nabla_{x}\left(D_{\theta}(r-s)\right), v=D_{\theta} s$, and $w=$ $\nabla_{y}\left(D_{\theta} s\right)$. The non-negative constraints in the Eq. (5) is implemented by using a projection strategy. For the convenience purpose, $D_{\theta}(r-$ $s), D_{\theta} s$, and $D_{\theta} r$ are represented as $r_{D_{\theta}}-$ 
$s_{D_{\theta}}, s_{D_{\theta}}$, and $r_{D_{\theta}}$, respectively. Therefore, the augmented lagrangian function is mathematically denoted in Eq. (7).

$$
\begin{gathered}
\zeta\left(u, v, w, s, p_{1}, p_{2}, p_{3}\right)=\lambda_{1}\|u\|_{1}+ \\
<p_{1}, \nabla_{x}\left(r_{D_{\theta}}-s_{D_{\theta}}\right)-u>+\frac{\beta_{1}}{2} \| \nabla_{x}\left(r_{D_{\theta}}-s_{D_{\theta}}\right)- \\
u\left\|_{2}^{2}+\lambda_{2}\right\| v\left\|_{1}+<p_{2}, s_{D_{\theta}}-v>+\frac{\beta_{2}}{2}\right\| s_{D_{\theta}}-v \|_{2}^{2}+ \\
\|w\|_{1}+<p_{3}, \nabla_{y} s_{D_{\theta}}-w>+\frac{\beta_{3}}{2}\left\|\nabla_{y} s_{D_{\theta}}-w\right\|_{2}^{2}
\end{gathered}
$$

Where, $p_{1}, p_{2}$, and $p_{3}$ are indicated as three Lagrange multipliers, and $\beta_{1}, \beta_{2}$, and $\beta_{3}$ are stated as regularization parameters. ADMM is a popular methodology to solve $l_{1}$ issue, and also it is utilized to solve four sub-problems such as u-subproblem, vsubproblem, w-subproblem, and $s_{D_{\theta}}$-subproblem.

\subsubsection{The u-subproblem}

$$
\begin{aligned}
& \hat{u}=\underset{u}{\operatorname{argmin}} \lambda_{1}\|u\|_{1}+<p_{1}, \nabla_{x}\left(r_{D_{\theta}}-s_{D_{\theta}}\right)- \\
& u>+\frac{\beta_{1}}{2}\left\|\nabla_{x}\left(r_{D_{\theta}}-s_{D_{\theta}}\right)-u\right\|_{2^{\prime}}^{2} \\
& =\underset{u}{\operatorname{argmin}} \lambda_{1}\|u\|_{1}+\frac{\beta_{1}}{2} \| \nabla_{x}\left(r_{D_{\theta}}-s_{D_{\theta}}\right)- \\
& u \frac{p_{1}}{\beta_{1}} \|_{2}^{2}
\end{aligned}
$$

The u-subproblem in Eq. (8) is solved precisely using soft-thresholding methodology that is mathematically denoted in Eq. (9).

$$
u^{k+1}=\operatorname{shrink}\left(\nabla_{x}\left(r_{D_{\theta}}-s_{D_{\theta}}^{k}\right)+\frac{p_{1}^{k}}{\beta_{1}}, \frac{\lambda_{1}}{\beta_{1}}\right)
$$

Where, $\operatorname{shrink}(a, b)=\operatorname{sign}(a) \max (|a|-b, 0)$ as mentioned in Eq. (10).

$$
\operatorname{sign}(a)= \begin{cases}1, & a>0 \\ 0, & a=0\end{cases}
$$

\subsubsection{The v-subproblem}

$$
\begin{aligned}
& \hat{v}=\underset{v}{\operatorname{argmin}} \lambda_{2}\|v\|_{1}+\left\langle p_{2}, s_{D_{\theta}}-v>+\right. \\
& \frac{\beta_{2}}{2}\left\|s_{D_{\theta}}-v\right\|_{2}^{2} \\
& =\underset{v}{\operatorname{argmin}} \lambda_{2}\|v\|_{1}+\frac{\beta_{2}}{2}\left\|s_{D_{\theta}}-v+\frac{p_{2}}{\beta_{2}}\right\|_{2}^{2}
\end{aligned}
$$

Henceforth, the closed form solution of $\mathrm{v}$ subproblem in Eq. (11) is achieved by utilizing soft thresholding strategy that is mathematically denoted in Eq. (12).

$$
v^{k+1}=\operatorname{shrink}\left(s_{D_{\theta}}^{k}+\frac{p_{2}^{k}}{\beta_{2}}, \frac{\lambda_{2}}{\beta_{2}}\right)
$$

\subsubsection{The w-subproblem}

$$
\begin{aligned}
& \widehat{w}=\underset{w}{\operatorname{argmin}}\|w\|_{1}+<p_{3}, \nabla_{y} s_{D_{\theta}}-w>+ \\
& \frac{\beta_{3}}{2}\left\|\nabla_{y} s_{D_{\theta}}-w\right\|_{2^{\prime}}^{2} \\
& =\underset{w}{\operatorname{argmin}}\|w\|_{1}+\frac{\beta_{3}}{2}\left\|\nabla_{y} s_{D_{\theta}}-w \frac{p_{3}}{\beta_{3}}\right\|_{2}^{2}
\end{aligned}
$$

The closed form solution of sub-problem (13) is mathematically denoted in Eq. (14).

$$
w^{k+1}=\operatorname{shrink}\left(\nabla_{y} s_{D_{\theta}}^{k}+\frac{p_{3}^{k}}{\beta_{3}}, \frac{1}{\beta_{3}}\right)
$$

\subsubsection{The $s_{D_{\theta}}$-subproblem}

$$
\begin{gathered}
\hat{s}_{D_{\theta}}=\underset{s_{D_{\theta}}}{\operatorname{argmin}}<p_{1}, \nabla_{x}\left(r_{D_{\theta}}-s_{D_{\theta}}\right)-u>+ \\
<p_{2}, s_{D_{\theta}}-v>+<p_{3}, \nabla_{y} s_{D_{\theta}}-w>+ \\
\frac{\beta_{1}}{2}\left\|\nabla_{x}\left(r_{D_{\theta}}-s_{D_{\theta}}\right)-u\right\|_{2}^{2}+ \\
\frac{\beta_{2}}{2}\left\|s_{D_{\theta}}-v\right\|_{2}^{2}+\frac{\beta_{3}}{2}\left\|\nabla_{y} s_{D_{\theta}}-w\right\|_{2}^{2} \\
=\underset{s_{D_{\theta}}}{\operatorname{argmin}} \frac{\beta_{1}}{2}\left\|\nabla_{x}\left(r_{D_{\theta}}-s_{D_{\theta}}\right)-u+\frac{p_{1}}{\beta_{1}}\right\|_{2}^{2}+ \\
\frac{\beta_{2}}{2}\left\|s_{D_{\theta}}-v+\frac{p_{2}}{\beta_{2}}\right\|_{2}^{2}+\frac{\beta_{3}}{2}\left\|\nabla_{y} s_{D_{\theta}}-w+\frac{p_{3}}{\beta_{3}}\right\|_{2}^{2}
\end{gathered}
$$

The quadratic $s_{D_{\theta}}$ - subproblem (15) is differentiable and the closed form solution is estimated by using the Eq. (16).

$$
\begin{aligned}
& \left.\beta_{1} \nabla_{x}^{T} \nabla_{x}+\beta_{2} I+\beta_{3} \nabla_{y}^{T} \nabla_{y}\right) s_{D_{\theta}}^{-k+1}= \\
& \nabla_{y}^{T}\left(\beta_{3} w^{k+1}-p_{3}^{k}\right)+\nabla_{x}^{T}\left(\beta_{1} \nabla_{x} r_{D_{\theta}}-\beta_{1} u^{k+1}-\right. \\
& \left.p_{1}^{k}\right)+\beta_{2} v^{k+1}-p_{2}^{k}
\end{aligned}
$$

Where, $I$ is indicated as identity matrix, and the Eq. (16) is solved effectively by utilizing Fast Fourier Transform (FFT). Because of non-negative constraint, the resultant $s_{D_{\theta}}^{-k+1}$ is estimated by using the Eq. (17).

$$
s_{D_{\theta}}^{-k+1}=\min \left(r_{D_{\theta}}, \max \left(s_{D_{\theta}}^{-k+1}, 0\right)\right)
$$

On the basis of ADMM, updated the Lagrange multipliers $p_{1}, p_{2}$, and $p_{3}$ by utilizing the Eq. (18). Therefore, the $v, u$ and $w$ subproblems are effectively solved by applying soft thresholding methodology, and $s_{D_{\theta}}$ subprobelm is computed by utilizing FFT algorithm. 


$$
\begin{aligned}
& p_{1}^{k+1}=p_{1}^{k}+\beta_{1}\left(\nabla_{x}\left(r_{D_{\theta}}-s_{D_{\theta}}^{k+1}\right)-u^{k+1}\right) \\
& p_{2}^{k+1}=p_{2}^{k}+\beta_{2}\left(s_{D_{\theta}}^{k+1}-v^{k+1}\right) \\
& p_{3}^{k+1}=p_{3}^{k}+\beta_{3}\left(\nabla_{y^{2}} s_{D_{\theta}}^{k+1}-w^{k+1}\right)
\end{aligned}
$$

As mentioned earlier, heavy rain generally incorporates haze and rain streaks, so the proposed DGSM cannot remove the rain streaks individually. So, a decomposition approach and Huber loss function are incorporated with DGSM for removing the rain streaks far away from the vertical direction. The SVD is an effective method that splits the system into a set of linearly independent components, which bears own energy contribution. The general equation of SVD is mathematically denoted in Eq. (19).

$$
A=u d v^{T}
$$

Where, $v$ is denoted as $n \times n$ orthogonal matrix, $u$ is indicated as $m \times n$ orthogonal matrix, $d$ is represented as $n \times n$ diagonal matrix, $A$ is stated as $m \times n$ matrix, $m$ is denoted as number of rows in the images, and $n$ is indicated as number of columns in the images. In addition, the Huber loss function generally utilizes in robust regression, which is less sensitive to outliers in the rainy images. The general equation of Huber loss function is specified in Eq. (20).

$$
L_{\delta}(R)=\left\{\begin{array}{cc}
\frac{1}{2} R^{2} & \text { for }|R| \leq \delta \\
\delta\left(|R|-\frac{1}{2} \delta\right) & \text { Otherwise }
\end{array}\right.
$$

Where, $\delta$ is represented as tunable parameter, and $R$ is indicated as rainy images. Eq. (21) represents the combination of SVD and Huber loss function need to be substituted in Eq. (18).

$$
A K 1=A+L_{\delta}(R)
$$

The Eq. (18) is updated as shown in Eq. (22). Respectively, Fig. 2 represent the both input and denoised images.

$$
\begin{aligned}
p_{1}^{k+1} & =p_{1}^{k}+\beta_{1}\left(\nabla_{x}\left(r_{D_{\theta}}-s_{D_{\theta}}^{k+1}\right)-u^{k+1}\right)- \\
A K 1 & p_{2}^{k+1}=p_{2}^{k}+\beta_{2}\left(s_{D_{\theta}}^{k+1}-v^{k+1}\right)-A K 1 \\
p_{3}^{k+1} & =p_{3}^{k}+\beta_{3}\left(\nabla_{y} s_{D_{\theta}}^{k+1}-w^{k+1}\right)-A K 1
\end{aligned}
$$
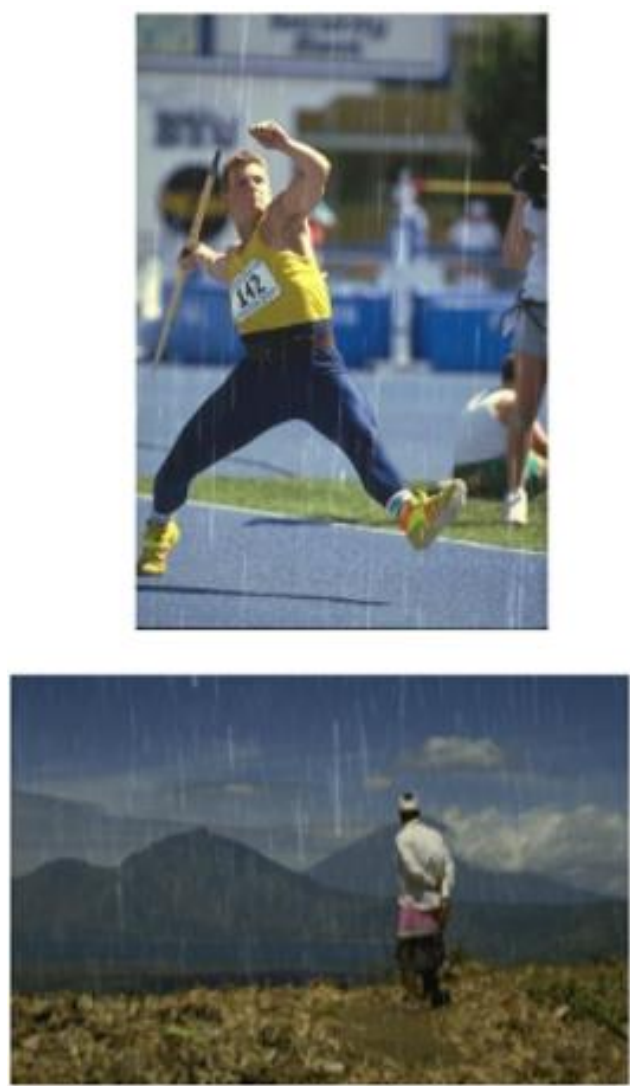

(a)
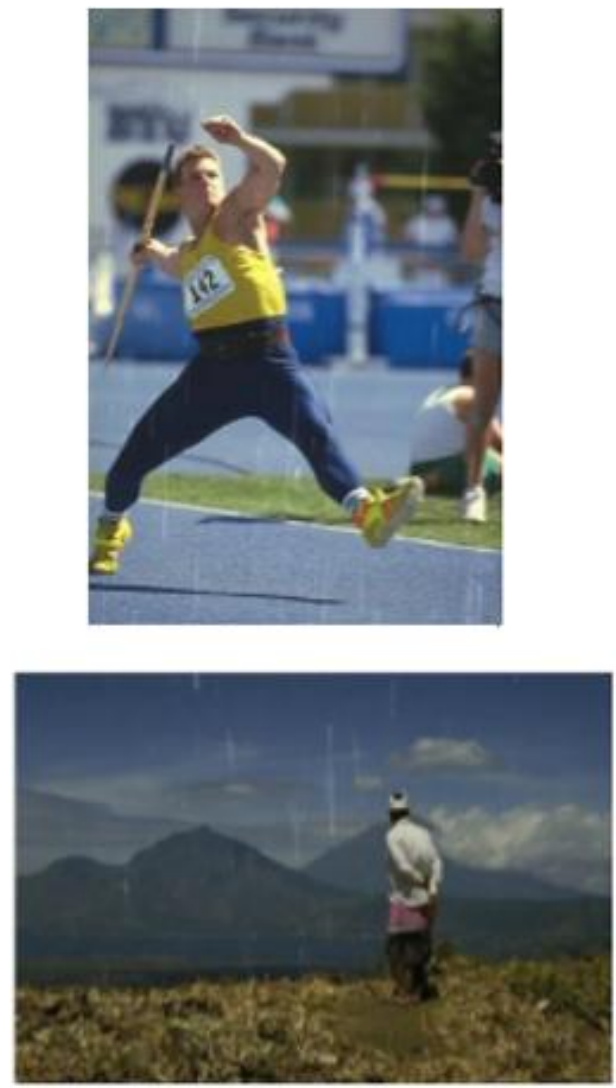

(b)

Figure.2 Sample images: (a) input image and (b) denoised image 


\section{Result and discussion}

In this paper, MATLAB (2018a) software was used for experimental simulation with Intel (R) Core (TM) i3-3220 CPU @ $3.30 \mathrm{GHz}$ and 8 GB (RAM). In the enhanced DGSM model, the parametric values were set as follows; $\lambda_{1}=0.95, \lambda_{2}=$ $0.08, \beta_{1}, \beta_{2}$ and $\beta_{3}=200$. In this research work, the enhanced DGSM model was compared with DGSM [16], and CAAN [17] to assess the efficacy of the proposed model. Here, the proposed work's performance was computed by using the performance metrics like SSIM, RMSE, and PSNR. The mathematical expression of SSIM, RMSE, and PSNR were represented in the Eq. (23), (24), and (25). The RMSE is a cumulative squared error between the original and denoised rainy colour image that is mathematically denoted in Eq. (23).

$$
R M S E=\sqrt{\frac{1}{N} \sum_{i=1}^{N}\left(h_{i}-\hat{h}_{i}\right)^{2}}
$$

Where, $\hat{h}_{i}$ is denoted as vector form of de-rain image, $h_{i}$ is represented as vector form of input or ground truth image, and $N$ is stated as number of image pixels. In addition, PSNR is utilized for measuring the quality of original and denoised rainy colour image that is mathematically denoted in Eq. (24).

$$
P S N R=10 \log _{10}\left(\frac{M A X_{1}^{2}}{M S E}\right)
$$

Where, $\quad M S E=\frac{1}{m n} \sum_{i=0}^{m-1} \sum_{j=0}^{n-1}[I(i, j)-$ $k(i, j)]^{2}, m \times n$ is indicated as monochrome image as $I, K$ is specified as noisy approximation, and $M A X_{1}$ is indicated as maximum possible pixel value of rainy image. The SSIM is used for measuring the similarity between the original and denoised rainy colour image, and it is mathematically stated in Eq. (25).

$$
\operatorname{SSIM}=\frac{\left(2 \mu_{x} \mu_{y}+C_{1}\right)\left(2 \sigma_{x y}+C_{2}\right)}{\left(\mu_{x^{2}}+\mu_{y^{2}}+C_{1}\right)\left(\sigma_{x^{2}}+\sigma_{y^{2}}+C_{2}\right)}
$$

Where, $\sigma_{y^{2}}$ is the variance of $y, \sigma_{x^{2}}$ is the variance of $x, \sigma_{x y}$ is the covariance of $x$ and $y, \mu_{y}$ is the average of $y$, and $\mu_{x}$ is the average of $x$.

\subsection{Quantitative analysis on synthesized images}

In this segment, three synthesized images (bamboo, panda, and tree) used for investigating the performance of the proposed model. In the Table 1 and 2, the proposed model performance is verified in terms of PSNR, RMSE, and SSIM. From the experimental study, the PSNR value of DGSM [16] for bamboo image is $30 \mathrm{~dB}$, and the proposed approach (enhanced DGSM) attained $31.009 \mathrm{~dB}$. Similarly, the PSNR value of DGSM [16] for panda image is $30.96 \mathrm{~dB}$, and the proposed model achieved $31.12 \mathrm{~dB}$. In addition, the PSNR value of DGSM [16] for tree image is $32.41 \mathrm{~dB}$, and the proposed model attained $32.93 \mathrm{~dB}$. In this consequence, the proposed enhanced DGSM model almost showed 0.5-1 dB improvement in PSNR compared to the existing model (DGSM [16]). Correspondingly, the RMSE value of DGSM [16] model for bamboo image is $7.227 \mathrm{~dB}$, and the proposed model achieved 7.17 error value. For panda image, the RMSE value of DGSM [16] is 7.219 and the enhanced DGSM model attained 7.08 error value. Similarly, in tree image, the RMSE value of DGSM [16] is 6.112 and the enhanced DGSM model attained 6.10 error value. The experimental analysis showed that the proposed model attained decent performance in terms of PSNR and RMSE. Graphical comparison of the proposed model in light of PSNR and RMSE on synthesized images is denoted in Fig. 3.

In Table 2, the performance investigation of the proposed model is done by means of SSIM value. From the inspection, the SSIM value of the enhanced DGSM of bamboo, panda, and tree images are highly related to the existing model (DGSM [16]). In this scenario, the proposed model almost showed 0.005 value improvement in SSIM compared to DGSM [16]. Graphical comparison of the proposed model in light of SSIM on synthesized images is denoted in Fig. 4.

Table 1. Performance analysis of the proposed model in light of PSNR and RMSE on synthesized images

\begin{tabular}{|c|c|c|c|c|}
\hline \multirow{2}{*}{ Images } & \multicolumn{2}{|c|}{ PSNR (dB) } & \multicolumn{2}{c|}{ RMSE } \\
\cline { 2 - 5 } & $\begin{array}{c}\text { DGSM } \\
{[\mathbf{1 6}]}\end{array}$ & $\begin{array}{c}\text { Enhanced } \\
\text { DGSM }\end{array}$ & $\begin{array}{c}\text { DGSM } \\
{[\mathbf{1 6}]}\end{array}$ & $\begin{array}{c}\text { Enhanced } \\
\text { DGSM }\end{array}$ \\
\hline Bamboo & 30.95 & 31.009 & 7.227 & 7.17 \\
\hline Panda & 30.96 & 31.12 & 7.219 & 7.08 \\
\hline Tree & 32.41 & 32.93 & 6.112 & 6.10 \\
\hline
\end{tabular}

Table 2. Performance analysis of the proposed model by means of SSIM on synthesized images

\begin{tabular}{|c|c|c|}
\hline \multirow{2}{*}{ Images } & \multicolumn{2}{|c|}{ SSIM } \\
\cline { 2 - 3 } & DGSM [16] & Enhanced DGSM \\
\hline Bamboo & 0.9176 & 0.9179 \\
\hline Panda & 0.9324 & 0.9328 \\
\hline Tree & 0.9358 & 0.9408 \\
\hline
\end{tabular}




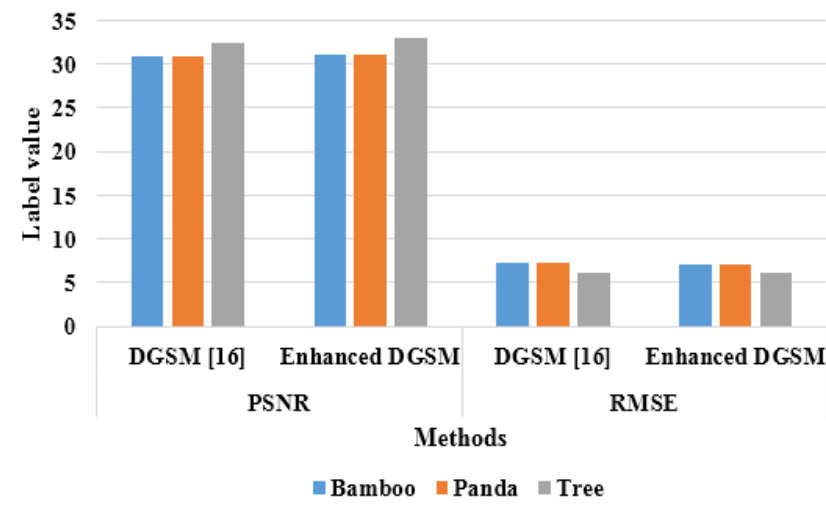

Figure. 3 Graphical comparison of the proposed model in light of PSNR and RMSE on synthesized images

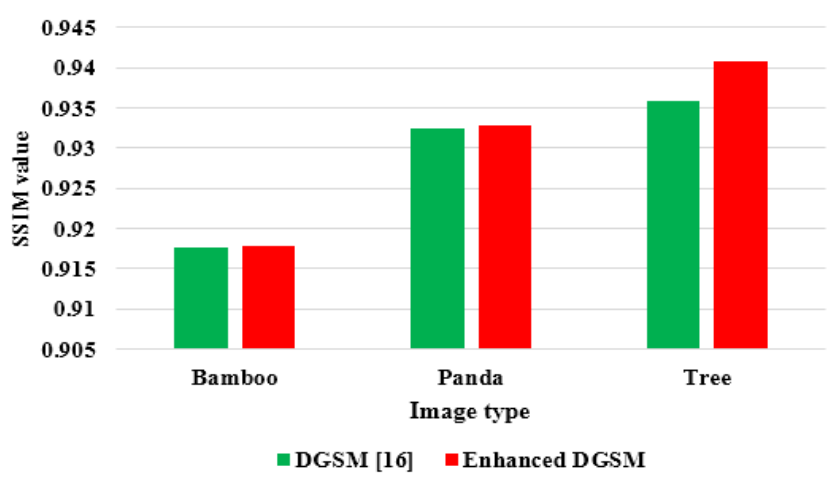

Figure.4 Graphical comparison of the proposed model by means of SSIM on synthesized images

In Table 3, the performance of the proposed model is analyzed for the remaining twelve images. In Table 3, the average PSNR value of DGSM [16] is $33.18 \pm 3.5 \mathrm{~dB}$, and the proposed model attained $33.55+5.1 \mathrm{~dB}$, which is almost $0.37 \mathrm{~dB}$ higher than the existing model. Likewise, the average SSIM value of DGSM [16] is $0.9365 \pm 0.02$, and the proposed model attained $0.9372+0.05$, which is 0.005 value greater than the existing model.
Table 3. Performance analysis of the proposed model by means of PSNR, SSIM, and RMSE on a new dataset

\begin{tabular}{|c|c|c|c|c|c|c|}
\hline \multirow{2}{*}{$\begin{array}{c}\text { Ima } \\
\text { ges }\end{array}$} & \multicolumn{2}{|c|}{ PSNR } & \multicolumn{2}{c|}{ SSIM } & \multicolumn{2}{c|}{ RMSE } \\
\cline { 2 - 7 } & $\begin{array}{c}\text { DGS } \\
\text { M } \\
\end{array}$ & $\begin{array}{c}\text { Enha } \\
\text { nced }\end{array}$ & $\begin{array}{c}\text { DGSM } \\
\text { DGS } \\
\text { [16] }\end{array}$ & $\begin{array}{c}\text { Enhan } \\
\text { ced } \\
\text { MGSM }\end{array}$ & $\begin{array}{c}\text { DGS } \\
\text { M } \\
{[16]}\end{array}$ & $\begin{array}{c}\text { Enha } \\
\text { nced } \\
\text { DGS } \\
\text { M }\end{array}$ \\
\hline Mea & 33.18 & 33.55 & 0.9365 & 0.9372 & 6.033 & $5.72+$ \\
n & \pm 3.5 & +5.1 & \pm 0.02 & +0.05 & \pm 2.4 & 3.01 \\
valu & & & & & & \\
e of & & & & & & \\
all & & & & & & \\
12 & & & & & & \\
ima & & & & & & \\
ges & & & & & & \\
\hline
\end{tabular}

Table 4. Performance analysis of the proposed model by means of PSNR, and SSIM on rain $100 \mathrm{H}$ and rain 100L datasets

\begin{tabular}{|c|c|c|c|c|}
\hline Datasets & \multicolumn{2}{|c|}{ Rain100H } & \multicolumn{2}{c|}{ Rain100L } \\
\hline Methods & SSIM & $\begin{array}{c}\text { PSNR } \\
\text { (dB) }\end{array}$ & SSIM & $\begin{array}{c}\text { PSNR } \\
\text { (dB) }\end{array}$ \\
\hline CAAN [17] & 0.820 & 24.03 & 0.971 & 36.25 \\
\hline $\begin{array}{c}\text { Enhanced } \\
\text { DGSM }\end{array}$ & 0.831 & 25.051 & 0.98 & 37.24 \\
\hline
\end{tabular}

In addition, the error value of proposed model is $5.72+3.01$, which is 0.31 value lower than the existing model (DGSM [16]). The major benefit of using enhanced DGSM is to effectively preserve the non-rain image pixel details and to remove the rain image pixels that helps in attaining better rain streak removal performance.

\subsection{Quantitative analysis on rain100H and rain100L datasets}

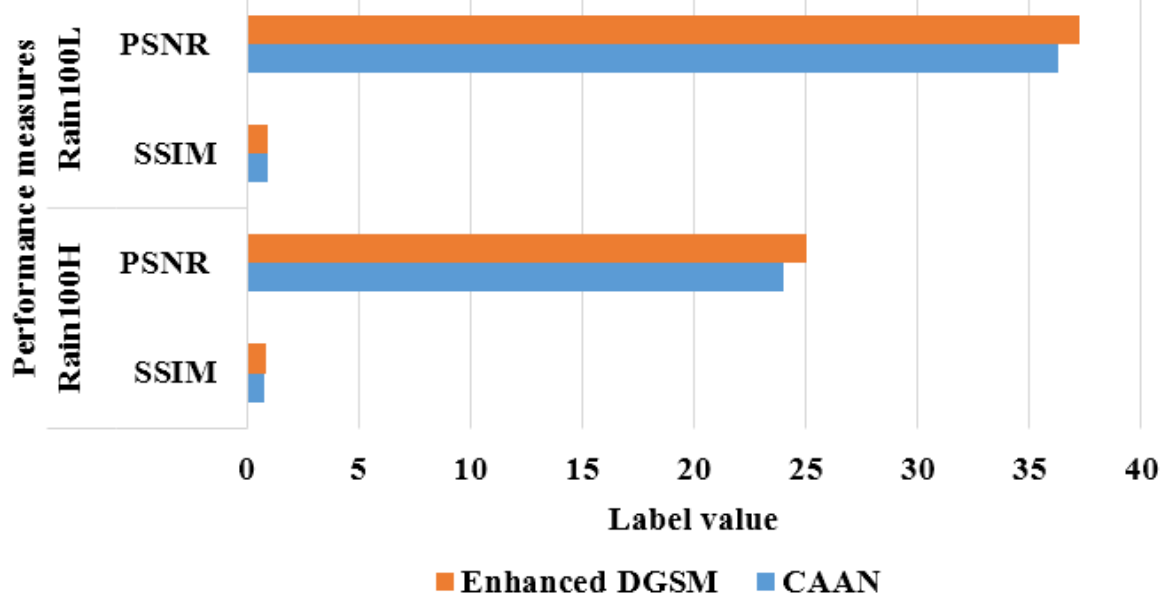

Figure.5 Graphical comparison of the proposed and existing model on rain100H and rain100L datasets 
In this segment, rain $100 \mathrm{H}$ and rain $100 \mathrm{~L}$ datasets used, to validate the performance of the proposed and existing model (CAAN [17]). In this scenario, the performance evaluation performed for 200 images in rain $100 \mathrm{H}$ dataset and 100 images in rain $100 \mathrm{~L}$ dataset. The validation consequence showed that the proposed model outperformed the existing model (CAAN [17]) in light of PSNR and SSIM. In rain $100 \mathrm{H}$ dataset, the proposed model showed 0.831 of SSIM and $25.051 \mathrm{~dB}$ of PSNR value, but the existing model obtained 0.820 of SSIM and $24.03 \mathrm{~dB}$ of PSNR value. Similarly, in rain $100 \mathrm{~L}$ dataset, the proposed model obtained 0.98 of SSIM and $37.24 \mathrm{~dB}$ of PSNR value, and the existing model attained 0.971 of SSIM and $36.25 \mathrm{~dB}$ of PSNR value. From the experimental analysis, the enhanced DGSM model outperformed the existing model (CAAN [17]) in terms of SSIM and PSNR. Graphical comparison of the proposed and existing model on rain $100 \mathrm{H}$ and rain100L datasets is denoted in Fig. 5.

\section{Conclusion}

The rain streaks not only decrease the image visibility and makes computer vision systems fail to function appropriately. In this work, a global sparse model with SVD and Huber loss function is employed for removing rain streaks from the synthesized color images. The proposed model uses three sparse terms for describing the intrinsic and latent properties of rain streaks, and also to smooth the rain free and streak image regions. In addition, $l_{1}$ norm was used to eliminate undesired features on the rainy free regions that helps in achieving better rain removal performance. Compared to the previous research works (DGSM, and CAAN), the proposed model attained good performance in light of RMSE, PSNR and SSIM value. The proposed model almost showed 0.52-1 dB enhancement in PSNR value. In future work, an optimization technique can be included in the proposed model to further remove the heavy rain streaks in the single colour image.

\section{References}

[1] J. Xiao, W. Zou, Y. Chen, W. Wang, and J. Lei, "Single image rain removal based on depth of field and sparse coding", Pattern Recognition Letters, Vol.116, pp.212-217, 2018.

[2] S. Li, W. Ren, J. Zhang, J. Yu, and X. Guo, "Single image rain removal via a deep decomposition-composition network",
Computer Vision and Image Understanding, Vol.186, pp.48-57, 2019.

[3] K. Javed, G. Hussain, F. Shaukat, and S.O. Hwang, "A neural network approach to remove rain using reconstruction and feature losses", Neural Computing and Applications, pp.1-10, 2019.

[4] Y. Wang, and T.Z. Huang, "A Tensor-Based Low-Rank Model for Single-Image Rain Streaks Removal", IEEE Access, Vol.7, pp.83437-83448, 2019.

[5] Y. Luo, J. Zhu, J. Ling, and E. Wu, "Fast removal of rain streaks from a single image via a shape prior", IEEE Access, Vol.6, pp.6006960078, 2018.

[6] P. Mu, J. Chen, R. Liu, X. Fan, and Z. Luo, "Learning Bilevel Layer Priors for Single Image Rain Streaks Removal”, IEEE Signal Processing Letters, Vol.26, No.2, pp.307-311, 2018.

[7] L.W. Kang, C.W. Lin, and Y.H. Fu, "Automatic single-image-based rain streaks removal via image decomposition", IEEE Transactions on Image Processing, Vol.21, No.4, pp.1742-1755, 2011.

[8] H. Tang, L. Zhu, D. Zhang, and X. Wang, "Single image rain removal model using pure rain dictionary learning", IET Image Processing, Vol.13, No.10, pp.1797-1804, 2019.

[9] X. Fu, J. Huang, D. Zeng, Y. Huang, X. Ding, and J. Paisley, "Removing rain from single images via a deep detail network", In: Proc. of International Conf. On Computer Vision and Pattern Recognition, pp.3855-3863, 2017.

[10] W. Yang, R.T. Tan, J. Feng, J. Liu, S. Yan, and Z. Guo, "Joint Rain Detection and Removal from a Single Image with Contextualized Deep Networks", IEEE transactions on pattern analysis and machine intelligence, pp.1-1, 2019.

[11] Y. Luo and J. Ling, "Single-image de-raining using low-rank matrix approximation", Neural Computing and Applications, pp.1-12, 2019.

[12] D.Y. Chen, C.C. Chen, and L.W. Kang, "Visual depth guided color image rain streaks removal using sparse coding", IEEE transactions on circuits and systems for video technology, Vol.24, No.8, pp.1430-1455, 2014.

[13] X. Fu, J. Huang, X. Ding, Y. Liao, and J. Paisley, "Clearing the skies: A deep network architecture for single-image rain removal", IEEE Transactions on Image Processing, Vol.26, No.6, pp.2944-2956, 2017.

[14] Z. Shi, Y. Li, C. Zhang, M. Zhao, Y. Feng, and B. Jiang, "Weighted median guided filtering 
method for single image rain removal", EURASIP Journal on Image and Video Processing, Vol.2018, No.1, pp.35, 2018.

[15] C.H. Yeh, C.Y. Lin, K. Muchtar, and P.H. Liu, "Rain streak removal based on non-negative matrix factorization", Multimedia Tools and Applications, Vol.77, No.15, pp.20001-20020, 2018.

[16] L.J. Deng, T.Z. Huang, X.L. Zhao, and T.X. Jiang, "A directional global sparse model for single image rain removal", Applied Mathematical Modelling, Vol.59, pp.662-679, 2018.

[17] J. Wang, X. Huang, and S. Gai, "Single Image Rain Removal via Cascading Attention Aggregation Network on Challenging Weather Conditions", IEEE Access, Vol.7, pp.178848178861, 2019.

[18] W. Yang, R.T. Tan, J. Feng, J. Liu, Z. Guo, and S. Yan, "Deep joint rain detection and removal from a single image", In: Proc. of the IEEE Conference on Computer Vision and Pattern Recognition, pp.1357-1366, 2017. 